\title{
Factores relacionados con la pobreza, la inseguridad alimentaria y estrategias de afrontamiento en municipios marginados de Puebla, México
}

\author{
Factors related to poverty, food insecurity and coping strategies \\ in marginalized municipalities of Puebla, Mexico
}

\author{
José Luis Jaramillo-Villanueva*, Venkatesh Gurusamy**, Leobardo \\ Jiménez Sánchez* y Daniel Claudio Martínez-Carrera* \\ *Colegio de Posgraduados en Ciencias Agrícolas, México \\ **JKK Munirajah College of Agricultural Science
}

\section{Resumen}

El objetivo de la presente investigación fue identificar y explicar los factores determinantes de la condición de pobreza e inseguridad alimentaria de hogares rurales en municipios del estado de Puebla. Los datos se obtuvieron a través de una encuesta sociodemográfica, utilizando un diseño de muestreo por conglomerados, con confiabilidad del 95 por ciento y precisión de ocho por ciento. Éstos se modelaron con regresión logística. Las variables significativas $(\mathrm{p} \leq 0.05)$ en explicar la pobreza son aquellas que tienen un carácter prolongado en sus efectos; la educación, la posesión de activos, tener ganado mayor, tierra agrícola (mayor a 5 ha.), el empleo no agropecuario, y recibir crédito productivo. Las variables que explican la inseguridad alimentaria parecen tener efectos de corto plazo en el acceso a los alimentos; transferencias asistenciales, la producción para autoconsumo, y recibir remesas. Las cifras de pobreza, inseguridad alimentaria y la proporción del ingreso para alimentación, son ligeramente mayores a las oficiales en 2014.

Palabras clave: Hogar rural, pobreza, inseguridad alimentaria, ingreso del hogar.

\section{Abstract}

The aim of this research was to identify and explain the determinants of the condition of poverty and food insecurity of rural households in municipalities in the state of Puebla. The data was obtained through a demographic survey using a statistical cluster sampling design with reliability of 95 per cent and accuracy of 10 per cent. Data was analyzed using a logistic regression model. Significant variables $(\mathrm{p} \leq 0.05)$ in explaining poverty are those that have long time and persistent effects, such as; education, asset ownership, livestock, agricultural land (more than five ha), nonagricultural employment, and productive credit. The variables that explain food insecurity seem to have short-term effects on access to food; welfare transfers, production for self-consumption, and remittances. The figures of poverty, food insecurity and the proportion of income for food, are slightly higher than the official in 2014.

Key words: Rural household, poverty, food insecurity, household income. 


\section{INTRODUCCIÓN}

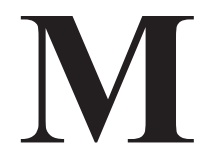

éxico tiene características ecológicas, climatológicas, culturales, sociales y económicas diversas, a lo largo y ancho del país. Esto le confiere una diversidad de formas para practicar una agricultura y ganadería altamente diversificada, capaz de producir todo el año. Las estadísticas oficiales registran el cultivo de 685 diferentes especies vegetales en forma anual o perenne, y la cría de siete especies ganaderas. Al año se cosechan más de 60 millones de toneladas de granos, frutas y hortalizas, en promedio anual, en el periodo de 2006 a 2012, se obtuvieron 189.5 millones de toneladas de alimentos agropecuarios y pesqueros, 7.6 por ciento más que el promedio obtenido entre 2001 y 2005. Los productos agrícolas promediaron 169.2 millones de toneladas, los pecuarios 18.5 millones de toneladas, y los pesqueros 1.7 millones de toneladas (Schwentesius y Ayala, 2014: 3). De acuerdo con la Organización Mundial de Comercio (OMC), esto lo posiciona en el lugar 12 a nivel mundial como productor y exportador de alimentos en general y cuarto en alimentos orgánicos.

No obstante, México es altamente dependiente para alimentar a su población, más aún, el grado de autosuficiencia ha bajado en forma continua desde los años ochenta. En el año 2014 se importaron, principalmente desde Estados Unidos, 38 por ciento de los cuatro granos más importantes, 92 por ciento de las oleaginosas y 45 por ciento de los cárnicos consumidos en el país (SIAP, 2015). La dependencia alimentaria promedio para 2015 fue de 38 por ciento (calculado con datos de SIAP, 2015), cifra muy alta comparada con el 10 por ciento de dependencia máximo recomendado por FAO (2015).

En este sentido, la dependencia alimentaria es un factor que obstaculiza el objetivo social de mejorar la seguridad alimentaria, principalmente por la continua y acentuada volatilidad de los precios de los alimentos observada en las últimas tres décadas. Un determinado nivel de volatilidad tiene un impacto mayor en un escenario de precios altos, y, por tanto, mayor impacto negativo sobre el poder adquisitivo de los países y de los hogares (HLPE, 2011: 9).

El concepto de seguridad alimentaria, inicialmente, describía si un país tiene acceso a la cantidad de alimento para cubrir los requerimientos de energía de la población. La seguridad alimentaria se lograba si se producían los alimentos que la población necesita, y si ésta tenía acceso a ellos. Para mediados de la década de 1970 se reconoció que el aspec- 
to central del concepto es el acceso a los alimentos por parte de toda la población, en cantidad y calidad suficientes para una vida saludable y productiva (Pinstrup-Andersen, 2009: 5).

En la cumbre mundial de la alimentación, de Roma en 1996, este concepto se amplió, y se incluyó el valor nutricional y las preferencias alimentarias, para quedar de la siguiente forma: "La seguridad alimentaria existe cuando todas las personas tienen, en todo momento, acceso físico y económico a suficientes alimentos inocuos y nutritivos para satisfacer sus necesidades alimenticias y sus preferencias alimentarias para una vida activa y saludable" (FAO, 2006). La seguridad alimentaria es un concepto multidimensional, y en su consecución intervienen factores como la producción y productividad agrícola, las condiciones ambientales, el acceso a medios de producción, el comercio, los ingresos de las personas, la calidad de los alimentos, las condiciones de sanidad, y la gobernabilidad, entre los más importantes. La definición plantea cuatro dimensiones primordiales de la seguridad alimentaria: La disponibilidad física de los alimentos; el acceso económico y físico a los alimentos; la utilización de los alimentos; y la estabilidad en el tiempo de las tres dimensiones anteriores (Urquia-Fernández, 2014: 93).

Siguiendo los planteamientos de FAO (2011), la disponibilidad física aborda lo correspondiente a la "oferta", y es una función del nivel de producción, de las existencias, y del comercio neto de alimentos. No obstante, una oferta adecuada de alimentos no garantiza la seguridad alimentaria a nivel de los hogares, la producción de alimentos es sólo uno de los diferentes medios con los que cuentan las personas para adquirir los alimentos que necesitan. El acceso se refiere a que las personas y hogares tienen los recursos suficientes para adquirir alimentos apropiados y una alimentación nutritiva, y refleja el lado de la demanda, la cual pone de manifiesto la distribución desigual de los alimentos entre los hogares y al interior de estos. El acceso acentúa los problemas para responder a los choques adversos, como los períodos de desempleo, los aumentos de precios o la pérdida de activos que producen medios de subsistencia. A través del lente del acceso, la relación estrecha de la seguridad alimentaria con la pobreza y con la privación de derechos económicos y sociales se hace más evidente (Barrett, 2010: 825).

La utilización refleja las preocupaciones nutricionales sobre si las personas y los hogares hacen buen uso de los alimentos a los que tienen acceso. Ésta se centra en una mayor atención a la calidad dietética, especialmente las deficiencias de micronutrientes asociadas con la ingesta inadecuada de 
minerales esenciales y vitaminas. La utilización se entiende como la forma en la que el cuerpo aprovecha los diversos nutrientes presentes en los alimentos. Esta dimensión es función del estado de salud de las personas, la higiene y el saneamiento, la calidad del agua, las prácticas de cuidado de la salud, y la calidad e inocuidad de los alimentos (Barrett, 2013: 10).

Finalmente, la expresión "todas las personas, en todo momento" constituye un elemento integral de la definición de la seguridad alimentaria, y lo que representa es vital para el logro de los objetivos nacionales en materia de seguridad alimentaria. La expresión "en todo momento" se refiere a la dimensión de estabilidad de la seguridad alimentaria. Dicha expresión resalta la importancia de reducir los efectos adversos en las otras tres dimensiones: disponibilidad, acceso y utilización de los alimentos (FAO, 2011).

La pobreza es un fenómeno ligado con el desarrollo de un país, tanto en términos sociales, económicos, como democrático, de tal forma que, si se tiene crecimiento económico, éste se acompañe de una adecuada distribución. Tasas elevadas de crecimiento económico, deben acompañarse de mayor equidad en la distribución de los recursos entre los individuos de una sociedad (Ponce-Sernicharo, 2012: 1). La condición de pobreza es un fenómeno mundial, que afecta a sectores importantes, principalmente de países subdesarrollados, y va a seguir existiendo a menos que se tomen medidas contundentes para hacerle frente y erradicarla (Huerta, 2012: 68). Por esta razón, una adecuada conceptualización de la pobreza, así como la identificación de las personas y hogares en esta condición, y las causas que la generan, son tareas inaplazables.

De acuerdo con Haughton and Khandker (2009: 2-3), la pobreza "es la privación pronunciada en el bienestar de las personas." El bienestar se puede pensar en términos del control sobre los medios y recursos necesarios para conseguir algo, por lo que la gente está mejor si tienen un mayor control sobre los recursos". Aquí la atención se centra en si los hogares o individuos tienen recursos para satisfacer sus necesidades, en términos de la comparación del ingreso o del consumo con algún umbral (línea de pobreza) definido por debajo del cual se considera que son pobres. Esta forma de abordar la pobreza se denomina enfoque monetario.

Un segundo enfoque es preguntar si las personas son capaces de obtener un determinado tipo de bien de consumo. Este enfoque se centra en conocer el acceso beneficioso de las personas a ciertos satisfactores como los alimentos, la salud, la educación, entre otros. Un tercer enfoque se denomina de "capacidades" que en opinión de varios autores (Bolvinik, Alkire, Banco Mundial), conceptualmente es el más completo. Este fue propuesto 
por Sen (1987: 250 y 1992: 234) quien postula que el bienestar proviene de la "capacidad" de funcionar en la sociedad. De tal suerte que la condición de pobreza se da cuando las personas carecen de capacidades (alimentación, salud, educación, vestido, libertad de expresión, entre otras), que no les permite funcionar satisfactoriamente en la sociedad. La pobreza puede entenderse como deprivación de capacidades. La "capacidad" representa las varias combinaciones de funcionamientos (las cosas que una persona puede hacer y ser) que la persona puede lograr. Un conjunto de funcionamientos refleja la libertad de la persona para elegir llevar un tipo de vida u otro. De acuerdo con Valdés (1998: 7), Sen es partidario de que todas las personas tengan las mismas capacidades para aprovechar las oportunidades, y su concepto desplaza el análisis de la igualdad de oportunidades hacia la igualdad de capacidades.

La problemática de la pobreza, la inseguridad alimentaria y sus factores explicativos son temas que han sido abordados desde la década de los ochenta del siglo XX en diferentes países. Ravallion y Jalan (1998) encontraron que los activos productivos tienen un efecto positivo en reducir la pobreza y que acceso a salud y nivel educativo están correlacionados de forma significativa con la reducción de pobreza crónica. En este mismo sentido, Yunez-Naude y Taylor (2001) encontraron que el nivel educativo y posesión de activos aumentan significativamente el ingreso, especialmente el ingreso no agropecuario. Los factores asociados a la reducción de la inseguridad alimentaria fueron documentados en diferentes autores (Mango et al., 2014; Chinnakali et al., 2014; Harris-Fry et al., 2015; y Malik, 1996) quienes encontraron que poseer tierra, mayor nivel educativo de la mujer, activos productivos y asistencia social reducen la inseguridad alimentaria, mientras que hogares con mayor número de integrantes menores de 12 años y escolaridad baja del jefe del hogar aumentan la probabilidad de padecer inseguridad alimentaria.

En Puebla, la población en condición de pobreza e inseguridad alimentaria, en términos porcentuales, es mayor que a nivel nacional. El CONEVAL (2016) reportó que, en 2014, la tasa de pobreza en áreas rurales fue del 61 por ciento a nivel nacional y de 65 por ciento en áreas rurales del estado de Puebla. La pobreza extrema y la pobreza por acceso a la alimentación, a nivel nacional, para áreas rurales fue de 9.5 por ciento y 19.7 por ciento respectivamente, en tanto que, para Puebla, fue de 16 por ciento y 24 por ciento respectivamente.

Un aspecto importante para el diseño y operación de intervenciones eficaces contra la pobreza es la identificación adecuada de las personas 
y hogares que padecen esta condición. Para mejorar los resultados de la lucha contra la pobreza y la inseguridad alimentaria, es apremiante un adecuado entendimiento de los factores explicativos asociados a estos fenómenos (Gundersen and Garasky, 2012: 1865). Las encuestas nacionales, principalmente la Encuesta Nacional de Ingreso y Gasto de los Hogares (ENIGH), no tiene representatividad municipal, por lo que los resultados agregados pueden enmascarar la situación intra municipal. En este sentido, los estudios regionales pueden aportar información detallada sobre las características de las personas y hogares en condición de pobreza, y sobre las variables asociadas a la pobreza, para una mejor comprensión del fenómeno, y eventualmente mejorar el diseño y operación de programas de intervención.

La condición de inseguridad alimentaria y pobreza son dos fenómenos estrechamente relacionados. El bienestar social, aproximado con el ingreso y con el consumo de los hogares rurales depende no solo del ingreso monetario, sino también del acceso a una serie de activos productivos, de las condiciones naturales y ambientales en que se da la reproducción de la vida social de este tipo de hogares, y del tipo de relaciones que se establecen entre ellos. La hipótesis que sustentamos es que la condición de pobreza e inseguridad alimentaria de los hogares rurales está relacionada con los niveles de ingreso y consumo, y explicada por características sociodemográficas, nivel educativo, composición de los hogares, y por el acceso a activos productivos.

\section{Metodología}

\section{Localización del área de estudio}

Las caracteristicas físicas de los tres municipios que integran la zona del estudio se obtuvieron de la enciclopedia de los municipios y delegaciones de México (INAFED, 2010) en tanto que las características de pobreza e inseguridad alimentaria, la fuente es CONEVAL (2016).

El municipio de San Salvador el Verde se localiza en la parte centro Oeste, el clima predominante es templado subhúmedo con lluvias en verano. Temperatura media anual entre 12 y 18 grados centígrados; las faldas inferiores de la Sierra Nevada están dedicas en su mayoría a la agricultura de temporal. Al oriente presenta áreas importantes de agricultura de riego. Datos de CONEVAL (2016) señalan que la pobreza alcanza a 71.1 por ciento de los hogares y la inseguridad alimentaria a 20.2. 
Factores relacionados con la pobreza, la inseguridad alimentaria y estrategias ... / J.L. JARAMILLO VILLANUEVA et al.

El municipio de Tlapanalá se localiza en la parte suroeste del Estado de Puebla, dentro de la zona de climas cálidos del Valle de Izúcar de Matamoros, identificándose dos climas: semicálido y cálido subhúmedo. La mayor parte del municipio presenta áreas dedicadas a las actividades agrícolas; existen grandes zonas de agricultura de temporal. La pobreza alcanza a 77.4 por ciento y la inseguridad alimentaria a 25.1 por ciento (CONEVAL, 2016).

Por su parte, el municipio de Coatzingo se localiza en la parte centro sur del estado de Puebla. Presenta un clima cálido subhúmedo con lluvias en verano. Existe un área importante dedicada a la agricultura de riego en la zona plana. El porcentaje de hogares en condiciones de pobreza en 2015 fue 85.2 y con inseguridad alimentaria fue de 25.1 (CONEVAL, 2016).

El diseño de muestreo fue por conglomerados en dos etapas. Se seleccionó este método porque no existe un marco de muestreo de hogares, pero si conocíamos las comunidades dentro de cada uno de los municipios seleccionados. En la primera etapa se seleccionaron tres municipios de forma no aleatoria. El criterio fue el contraste detectado en recorridos de campo, en sus características productivas, sociodemográficas y climáticas, en congruencia con la hipótesis planteada. En una segunda etapa, se realizó un muestreo aleatorio simple; a partir de la lista de comunidades de cada municipio. A partir de la población total de los tres municipios (9042 hogares), se calculó el tamaño de muestra, usando la fórmula de varianza máxima, que arrojó una muestra de 141 hogares, cantidad que se distribuyó proporcionalmente al tamaño de cada municipio (según número de hogares) (Tabla 1).

Tabla 1: Población, muestra y distribución de los hogares seleccionados

\begin{tabular}{lrrrr}
\hline Municipio & $\begin{array}{r}\text { Núm. de } \\
\text { hogares }\end{array}$ & Población total & Porcentaje & Muestra \\
\hline San Salvador & 6459 & 28419 & 0.70 & 98 \\
el Verde & 2050 & 8404 & 0.22 & 31 \\
Tlapanalá & 741 & 2964 & 0.08 & 11 \\
Coatzingo & 9250 & 39787 & 1.00 & 141 \\
& & & & \\
\hline
\end{tabular}

Fuente: INEGI (2014).

Para la colecta de datos se aplicó una encuesta socioeconómica. La prueba piloto y la aplicación de la encuesta se realizaron de septiembre a noviembre de 2014, con la participación de cuatro profesionistas previamente capacitadas. En esta etapa se realizaron tres acciones simultánea- 
mente; se levantó la encuesta piloto, se corrigió el cuestionario, se capacitó a los encuestadores, y se levantó la encuesta, consistente en 141 entrevistas a jefes(as) de hogar.

\section{Las variables del estudio}

Las variables principales que se analizaron en este estudio se describen en la Tabla 2. El primer grupo se refiere a las características sociodemográficas, el segundo a las características del hogar y el tercero a los activos y tipo de ingreso del hogar. Las dos variables más relevantes son pobreza e inseguridad alimentaria. Para pobreza se utilizó el concepto de "pobreza por ingresos" y se aproximó con el ingreso corriente del hogar. Se generaron dos niveles: pobres y no pobres. Los primeros tuvieron ingreso inferior al necesario para cubrir la canasta alimentaria y no alimentaria. Los segundos, su ingreso fue igual o mayor que el necesario para cubrir ambas canastas. Para inseguridad alimentaria se utilizó la escala de inseguridad alimentaria, que considera tres niveles; leve, moderada y severa. Las variables explicativas utilizadas en los modelos econométricos son las reportadas en estudios previos (Scott y Diwakar, 2016; Ravallion y Jalan, 1998; Magaña-Lemus et al., 2016; Yunez-Naude y Taylor, 2001; Harris-Fry et al., 2015; y Malik, 1996).

\section{Concepto de ingreso del hogar}

Con el propósito de hacer comparables los resultados de esta investigación con otros reportados en la literatura, se utilizó el concepto de ingreso que propone INEGI (2015), y que es el mismo que utiliza CONEVAL en las mediciones de bienestar y pobreza en México. El concepto de ingreso es el que se forma por las entradas monetarias y no monetarias que satisfacen tres criterios: regularidad, disponibilidad y patrimonio. El ingreso corriente, de acuerdo con INEGI, se compone de las siguientes cinco categorías: Ingreso del Trabajo; Renta de la Propiedad; Transferencias; Estimación del Alquiler de la Vivienda; y Otros ingresos corrientes. Dada las características del estudio y por cuestiones de costos no fue posible imputar un valor para el alquiler de la vivienda, ni de renta de la propiedad, por lo que, por simplicidad no se tomaron en cuenta para las estimaciones de ingreso corriente. 
Factores relacionados con la pobreza, la inseguridad alimentaria y estrategias ... / J.L. JARAMILLO VILLANUEVA et al.

Tabla 2: Variables utilizadas en el análisis de pobreza e inseguridad alimentaria

\begin{tabular}{|c|c|}
\hline Nombre de la variable & Concepto y escala de medición \\
\hline Genero del jefe(a) & $\begin{array}{l}\text { Si el jefe(a) del hogar es hombre o mujer, medida en escala } \\
\text { nominal; } \\
\text { hombre }=0 ; \text { Mujer }=1\end{array}$ \\
\hline Edad del jefe(a) & Número de años cumplidos. Escala de intervalo \\
\hline Escolaridad del jefe(a) & $\begin{array}{l}\text { Número de años de escolaridad, sin considerar preescolar. } \\
\text { Escala de intervalo }\end{array}$ \\
\hline Razón de dependencia & $\begin{array}{l}\text { Número de personas que no se encuentra en edad productiva } \\
\text { (menores de } 15 \text { años y mayores de } 65 \text { ) entre el número de } \\
\text { personas productivas. Escala de intervalo. }\end{array}$ \\
\hline Habla lengua indígena & $\begin{array}{l}\text { Si el jefe(a) del hogar habla lengua indígena. Escala } \\
\text { nominal; } \\
\text { No }=0 ; \text { Sí }=1\end{array}$ \\
\hline Ocupación principal & $\begin{array}{l}\text { La ocupación productiva que genera mayor ingreso } \\
\text { monetario. Escala nominal; }\end{array}$ \\
\hline Recibe programas asistenciales & $\begin{array}{l}\text { Si el hogar recibió transferencias de tipo asistencial } \\
\text { Escala nominal; } \mathrm{No}=0 ; \mathrm{Si}=1\end{array}$ \\
\hline Tiene seguridad social & $\begin{array}{l}\text { Si el jefe(a) tiene acceso a servicios de salud } \\
\text { Escala nominal; } \mathrm{No}=0 ; \mathrm{Si}=1\end{array}$ \\
\hline Recibió remesas & $\begin{array}{l}\text { Si el hogar recibió remesas el año de la entrevista } \\
\text { Escala nominal; agropecuario; comercio, oficios, empleado }\end{array}$ \\
\hline Tiene parcelas agrícolas & $\begin{array}{l}\text { Si el hogar posee o trabaja parcelas agrícolas } \\
\text { Nominal; No }=0 ; \mathrm{Si}=1\end{array}$ \\
\hline Superficie sembrada & $\begin{array}{l}\text { Número de hectáreas sembradas con cultivos agrícolas } \\
\text { Escala de intervalo; ha. }\end{array}$ \\
\hline Unidades Animal (UA) & $\begin{array}{l}\text { Número de Unidades Animal; una vaca gestante y lactante } \\
\text { con un peso aproximado entre } 454 \mathrm{~kg} \text {. Se considera como } \\
\text { el animal básico para establecer equivalencias con otros } \\
\text { animales con relación al consumo de materia seca por día } \\
\text { o por año (Scarnecchia y Gaskins, 1987: 22-23). Escala de } \\
\text { intervalo }\end{array}$ \\
\hline Tiene servicios financieros & $\begin{array}{l}\text { Si el hogar recibió crédito productivo } \\
\text { Escala nominal; No }=0 ; \text { Sí }=1\end{array}$ \\
\hline Índice relativo de activos & $\begin{array}{l}\text { Construido a partir del número de activos productivos } \\
\text { del hogar (entre } 0 \text { y } 10 \text { activos). Escala ordinal; No tiene } \\
\text { activos }=0 ; 1-2 \text { activos }=0.2 ; 3-4=0.40 ; 5-6=0.60 ; 7-8= \\
0.80 ; 9-10=1.0\end{array}$ \\
\hline Realiza trabajo no agropecuario & $\begin{array}{l}\text { Si el jefe(a) del hogar realiza trabajo no agropecuario. } \\
\text { Escala nominal; No }=0 ; \mathrm{Sí}=1\end{array}$ \\
\hline Ingreso corriente & Ingreso Corriente (ver concepto): Escala de intervalo; pesos \\
\hline
\end{tabular}

Fuente: elaboración con datos de encuesta de campo, 2014. 


\section{La Escala del Componente de Acceso de la Inseguridad Alimentaria en el Hogar (HFIAS)}

Debido a que el concepto de seguridad alimentaria es un concepto complejo y multidimensional, la medición de la inseguridad alimentaria ha sido un reto constante tanto para los investigadores como para los tomadores de decisiones. Debido a esto, Coates et al. (2007: 2-3) propusieron indicadores relativamente simples, pero metodológicamente rigurosos del componente de acceso de la inseguridad alimentaria en el hogar que puedan utilizarse para guiar, controlar y evaluar intervenciones. Siguiendo la propuesta de Coates et al. (2007), la Escala de Acceso de la Inseguridad Alimentaria en el Hogar (HFIAS) se basa en la idea de que la experiencia de la inseguridad alimentaria (en lo que respecta al acceso) origina reacciones y respuestas predecibles que se pueden capturar y cuantificar a través de un estudio y resumir en una escala. La escala está formada por dieciocho preguntas, en las que se pide a las personas entrevistadas que describan el comportamiento y las actitudes, también denominados "dominios", de la experiencia de la inseguridad alimentaria. Los puntos límite de la escala permiten clasificar en términos categóricos si los hogares gozan o no de seguridad alimentaria. La ventaja de utilizar esta escala, con datos de México, es que se pueden hacer comparaciones con estudios en otras latitudes, tiene la desventaja de no ser completamente comparable con los estudios en México.

\section{Categorías de la escala}

(i) Un hogar con seguridad alimentaria no experimenta ninguna de las condiciones de inseguridad alimentaria (en lo que respecta al acceso) o sólo experimenta el sentimiento de preocupación, pero muy pocas veces. (ii) Un hogar con inseguridad alimentaria (en lo que respecta al acceso) leve se preocupa por no tener suficientes alimentos a veces o con frecuencia y/o no puede comer alimentos deseados y/o come una dieta más monótona que la deseada y/o algunos alimentos considerados no deseables, pero sólo muy pocas veces. Sin embargo, no reduce la cantidad ni experimenta ninguna de las tres condiciones más críticas (falta total de alimentos, irse a la cama con hambre o pasarse todo el día sin comer). (iii) Un hogar en un estado de inseguridad alimentaria moderada sacrifica la calidad con más frecuencia, comiendo una dieta monótona o alimentos menos apetecibles algunas veces o con frecuencia y/o ha comenzado a reducir la cantidad reduciendo el tamaño o número de las comidas, casi nunca o algunas veces. Sin embargo, no experimenta ninguna de las tres condiciones más severas. (iv) Un hogar 
Factores relacionados con la pobreza, la inseguridad alimentaria y estrategias ... / J.L. JARAMILLO VILLANUEVA et al.

con inseguridad alimentaria severa ha pasado a reducir el tamaño de las comidas o el número de comidas con frecuencia y/o experimenta cualquiera de las tres condiciones más severas (falta total de alimentos, irse a la cama con hambre o pasarse todo el día sin comer), incluso tan pocas veces como casi nunca. En otras palabras, cualquier familia que experimente una de estas tres condiciones incluso una sola vez en las últimas cuatro semanas (30 días) se considera que padece un severo estado de inseguridad alimentaria. De acuerdo con lo anterior, la inseguridad alimentaria de los hogares se midió utilizando la escala HFIAS, que es la base de la mayor parte de escalas cualitativas para medir inseguridad alimentaria en el mundo (Coates et al., 2006: 1438).

\section{El modelo económico y econométrico}

El modelo empírico es especificado de la siguiente forma:

$$
\mathrm{Yi}=(\mathrm{I}, \mathrm{H}, \mathrm{A}), \mathrm{i}=1,2, \ldots, \mathrm{n}
$$

Donde $Y$ es la variable dependiente que refleja la característica en turno (ser un hogar en condiciones de pobreza/inseguridad alimentaria). Las variables explicativas se derivan de las consideraciones teóricas descritas anteriormente y se agrupan en características individuales (I), características del hogar (H), y activos (A).

La estimación del modelo empírico se realizó usando el modelo logit binomial, para modelar pobreza y logit binomial múltiple para el modelo de inseguridad alimentaria. El modelo permite relacionar variables categóricas considerando una en particular como dependiente de las demás, a través de funciones de regresión (Jobson, 1992: 321). De acuerdo con Lobos y Viviani (2008: 69-70), si se define $P\left(Y=j / X_{i}\right)$ como la probabilidad condicionada de observar la categoría $j$ dado un conjunto de variables explicativas Xi ( $\mathrm{i}=1,2, \ldots, \mathrm{k})$, entonces si existen $j$ categorías de respuesta $(\mathrm{j}=0,1,2, \ldots, \mathrm{J})$ habrá $J$ ecuaciones que se debería resolver. Por lo tanto, $P\left(y=j / x_{i}\right)$ es una función de las combinaciones lineales $\beta_{j}{ }_{j} X$, donde $\beta$, es el vector de parámetros y $X$ es el vector de regresores. La ecuación del modelo de regresión logit binomial se puede generalizar para $k$ variables independientes, lo cual permite obtener la ecuación del modelo de regresión logit binomial múltiple, esto es:

$$
P(y=1)=\frac{1}{1+e^{-\beta^{\prime} x}}+\varepsilon_{i}=\frac{e^{\beta^{\prime} x}}{1+e^{\beta^{\prime} x}}+\varepsilon_{i}=\Lambda\left(\beta^{\prime} x\right)+\varepsilon_{i}
$$


Donde $p$ es la probabilidad del proceso binomial, $\Lambda\left(\beta^{\prime} x\right)$ es la función de distribución de probabilidad logística y el término aleatorio $\varepsilon_{i}$ (ruido blanco) sigue una distribución logística con media cero y varianza $\pi^{2} / 3$ (Greene, 2002: 667). Los parámetros del modelo logit son estimados por el método de máxima verosimilitud. El efecto marginal del modelo logit se obtiene como:

$$
\frac{\delta E(y / x)}{\delta \beta}=\boldsymbol{\Lambda}\left(\boldsymbol{\beta}^{\prime} x\right)\left(1-\boldsymbol{\beta}^{\prime} x\right)-\boldsymbol{\beta}
$$

La variable dependiente en el modelo de inseguridad alimentaria fue "inseguridad alimentaria" con tres niveles; leve, moderada, y severa. En el modelo de pobreza, la variable dependiente fue "Pobreza", con dos niveles; pobres y no pobres.

Como medidas de la bondad de ajuste del modelo, en este trabajo se usó el $\mathrm{R}^{2}$ ajustado de Nagelkerke (Pseudo- $\mathrm{R}^{2}$ ), la prueba de Hosmer-Lemeshow, y el estadístico de la razón de verosimilitud (-2 log de verosimilitud). Para medir la significancia estadística de los coeficientes del modelo (de las variables explicativas) se utilizó el estadístico de Wald.

\section{RESULTADOS Y DisCUSIÓN}

\section{Características de los hogares}

La muestra estuvo integrada por 141 jefes(as) de hogares rurales, de los cuales 72 por ciento tiene jefatura masculina y 28 por ciento femenina. El dato anterior es consistente con lo reportado por Pedrero (2004: 432) e INEGI (2014) quienes reportaron valores de 21.7 por ciento y 25 por ciento para el país y Puebla, respectivamente. El ocho por ciento de los jefes(as) hablan lengua indígena. La escolaridad promedio del jefe(a) es de 7.3 años; 6.0 si es mujer y 7.8 si es hombre. Entre municipios, la escolaridad del jefe(a) es mayor para San Salvador el Verde, con 9.8 años. El promedio de integrantes del hogar es de 4.1, cifra muy similar al promedio del estado, de 4.16 (INEGI, 2014). La razón de dependencia promedio es de 0.68 , es decir que 68 de cada 100 personas son dependientes económicamente. Este valor es mayor que el promedio nacional y estatal, que son de 0.60 y 0.66 respectivamente (CONAPO, 2012).

Las transferencias y los servicios que reciben los hogares juegan un papel importante en mitigar los efectos de la pobreza y la inseguridad alimentaria de los hogares marginados. Al respecto se encontró que 34.8 por 
Factores relacionados con la pobreza, la inseguridad alimentaria y estrategias ... / J.L. JARAMILLO VILLANUEVA et al.

ciento de los hogares recibe transferencias monetarias de algún programa de gobierno, principalmente "Prospera" y "Proagro". Sobre este aspecto, Sadoulet et al. (2001: 1049), encontraron que las transferencias en efectivo generaron multiplicadores del ingreso en el rango de 1.5 a 2.6 en los hogares beneficiarios, lo que, a su vez, propició aumentos en la demanda efectiva.

Sobre los servicios sociales, se tiene que 49 por ciento de los hogares tiene acceso a servicios de salud; 32.1 por ciento tiene seguro popular y 16.9 por ciento tiene atención de diferentes instituciones: Instituto Mexicano del Seguro Social (IMSS) e Instituto de Seguridad y Servicios Sociales de los Trabajadores del Estado (ISSSTE), y servicios privados. El 15.5 por ciento recibe remesas entre tres y ocho veces al año, y 16.3 por ciento utiliza servicios financieros de crédito y 13.5 por ciento de ahorro, principalmente de microfinancieras y cajas de ahorro. Con respecto a las actividades productivas y los activos, la ocupación principal, de la que el hogar obtiene la mayor parte de sus recursos para vivir, es la agricultura y ganadería para 63.1 por ciento de los jefes(as), y las actividades no agropecuarias para 36.9 por ciento. Estos valores son más bajos que los reportados por De Janvry y Sadoulet (2001: 1047), quienes, en un estudio en México, encontraron que las Unidades de Producción Rural (UP) obtienen 55 por ciento del total del ingreso del hogar, de realizar actividades no agropecuarias. Entre municipios, llama la atención San Salvador el Verde, donde 56 por ciento de los hogares tienen como principal fuente de ingresos las actividades no agropecuarias. Una posible explicación es su cercanía a las zonas urbanas y a su mayor nivel educativo. Rahut y Micevska (2012: 574) y Jonasson y Helfand, (2010: 12) reportaron que los años de escolaridad y la distancia de la comunidad a los centros urbanos son significativas en explicar la selección de actividades no agropecuarias.

El 69.8 por ciento de los hogares tienen parcelas agrícolas, con un tamaño de 3.1 hectáreas, de las cuales, siembran en promedio 2.1 hectáreas, principalmente de maíz, frijol, frutales caducifolios y flores. Los datos anteriores son similares a los reportados por INEGI (2007), quien reporto que 83 por ciento de las UP en Puebla tiene superficies menores a cinco hectáreas. Entre municipios, los hogares de San Salvador el Verde detentan en promedio, una mayor cantidad de tierra agrícola, sin embargo, siembran poco más de 50 por ciento de esta superficie. Los otros dos municipios siembran casi toda la superficie que poseen. Coatzingo tiene un mayor número de UP, en tanto que San Salvador el Verde posee el mayor número 
de activos productivos, con un promedio de 0.5 , es decir cinco activos, de un total de diez posibles.

\section{Estructura del ingreso y del consumo}

Teórica y empíricamente, los niveles de ingreso y consumo están asociados con el nivel de pobreza y del nivel de inseguridad alimentaria de los hogares (Wang et al., 2016: 13-14; Alkire y Foster, 2011: 478). Los resultados encontrados están en esta dirección. Los municipios de Tlapanalá y Coatzingo tienen menores niveles de ingreso y consumo que San Salvador el Verde, también presentan mayores niveles de pobreza e inseguridad alimentaria (Tabla 3). En general, los tres municipios presentan mayores niveles de pobreza e inseguridad alimentaria que los reportados por CONEVAL (2016) para población rural en el estado de Puebla.

Tabla 3: Niveles de seguridad e inseguridad alimentaria a nivel municipal y región

\begin{tabular}{|c|c|c|c|c|c|c|c|c|}
\hline \multirow{2}{*}{ Niveles } & \multicolumn{2}{|c|}{$\begin{array}{l}\text { San Salvador } \\
\text { el Verde }\end{array}$} & \multicolumn{2}{|c|}{ Tlapanalá } & \multicolumn{2}{|c|}{ Coatzingo } & \multicolumn{2}{|c|}{ General } \\
\hline & Frec. & $\%$ & Frec. & $\%$ & Frec. & $\%$ & Frec. & $\%$ \\
\hline Seguridad & 45 & 46.88 & 14 & 37.84 & 3 & 37.50 & 62 & 43.97 \\
\hline Inseguridad leve & 23 & 23.96 & 11 & 29.73 & 1 & 12.50 & 35 & 24.82 \\
\hline $\begin{array}{l}\text { Inseguridad } \\
\text { moderada }\end{array}$ & 17 & 17.71 & 7 & 18.92 & 2 & 25.00 & 26 & 18.44 \\
\hline $\begin{array}{l}\text { Inseguridad } \\
\text { severa }\end{array}$ & 11 & 11.46 & 5 & 13.51 & 2 & 25.00 & 18 & 12.77 \\
\hline Total & 96 & 100.00 & 37 & 100.00 & 8 & 100.00 & 141 & 100.00 \\
\hline Pobreza & 66 & 68.75 & 27 & 72.97 & 6 & 75 & 99 & 70.21 \\
\hline
\end{tabular}

Fuente: elaboración con datos de encuesta de campo, 2014.

La condición de pobreza e inseguridad alimentaria está presente en 70 y 56 por ciento de los hogares. Estos niveles son más altos que el promedio estatal, que son de 65 y 50 por ciento, respectivamente (CONEVAL, 2016). Entre municipios, tanto la pobreza como la inseguridad alimentaria están presente en mayores tasas en Coatzingo y Tlapanalá, y en menor medida en San Salvador el Verde.

La principal diferencia entre los hogares urbanos y los rurales, en términos de su estrategia de sobrevivencia, es la diversificación del ingreso, esto es particularmente cierto en países con un nivel intermedio de desarrollo como el de México, con un sector agropecuario muy heterogéneo en términos del tamaño de las unidades de producción y del nivel tecnológico que utilizan. Los hogares rurales pequeños y aún los de tamaño medio 
Factores relacionados con la pobreza, la inseguridad alimentaria y estrategias ... / J.L. JARAMILLO VILLANUEVA et al.

producen cultivos básicos tanto para autoconsumo, como para vender excedentes al mercado, y perciben ingreso de empleos no agropecuarios (Yúnez-Naude y Taylor, 2004: 237).

El ingreso de los hogares estuvo integrado por siete conceptos, cuatro de naturaleza agropecuaria, transferencias y remesas, y dos de naturaleza no agropecuaria. A nivel regional, el ingreso agropecuario (IA) y el ingreso no agropecuario (INA) representaron 39 y 61 por ciento del ingreso total de los hogares, respectivamente (Tabla 4). Yúnez-Naude y Taylor, (2004: 237), en un estudio en México, realizado en ocho comunidades rurales, de los estados de Coahuila, Michoacán, Jalisco, y Puebla, encuentran que el empleo no agropecuario contribuyo con 60 por ciento del ingreso de los hogares.

Los componentes del ingreso agropecuario más importantes son la agricultura, que contribuyó con 16.5 por ciento, las transferencias y remesas contribuyeron con 14.5 por ciento. El ingreso no agropecuario salarial contribuye con 37 por ciento al ingreso total de los hogares. Entre municipios, se observa que para Coatzingo, el ingreso agropecuario es el más importante, con una contribución de 61.5 por ciento, en tanto que para San Salvador el Verde, este tipo de ingreso representa 40 por ciento, en cambio el ingreso no agropecuario contribuye con 60.3 por ciento.

Una explicación de lo anterior podría ser la cercanía de este último municipio a zonas urbanas de San Martín Texmelucan y la ciudad de Puebla que ofrecen una amplia gama de oportunidades en la industria y los servicios. Adicional a lo anterior, sabemos que los hogares en San Salvador el Verde tienen un mayor nivel educativo y, a pesar de detentar mayor superficie de tierra, siembran una superficie menor, posiblemente debido a que existe poca disponibilidad de mano de obra en la región, tanto familiar como contratada, que encuentra mejores salarios en empleos no agropecuarios.

\section{Estructura del consumo de los hogares}

Respecto al gasto (consumo) de los hogares, este se distribuye en dos grandes rublos, gasto alimentario y gasto no alimentario, que representan 47.1 y 52.9 por ciento, respectivamente, del gasto total del hogar. El gasto alimentario (GA) no incluyó el gasto en bebidas alcohólicas. El GA es mayor que el reportado por INEGI (2014) para áreas rurales de México, en tres puntos porcentuales. 


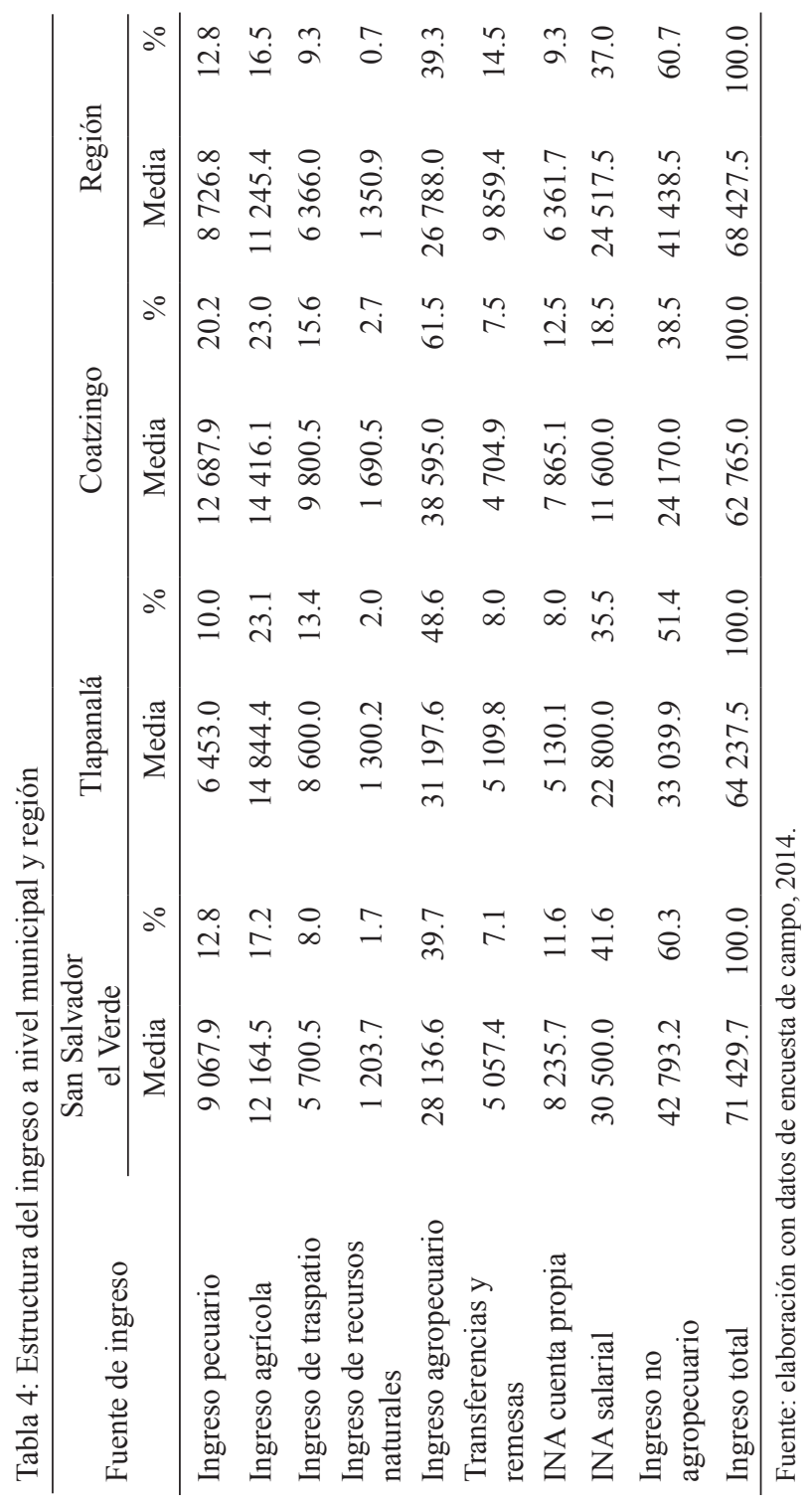


Factores relacionados con la pobreza, la inseguridad alimentaria y estrategias ... / J.L. JARAMILLO VILLANUEVA et al.

Este dato es consistente con el hecho de que los hogares rurales de este estudio presentan niveles más altos de pobreza, por lo que destinan una mayor proporción de su ingreso a la compra de alimentos. Dentro del gasto alimentario, los dos rublos más importantes son el grupo de "pan, tortilla y cereales" y "carnes lácteos y huevo", con 30.9 y 27.1 por ciento, respectivamente (Tabla 5).

Para estos mismos grupos, INEGI (2014) reportó un gasto de 22 y 36 por ciento, respectivamente. Lo anterior muestra que los hogares estudiados consumen más calorías, a partir de granos, y menor cantidad de proteína. El gasto no alimentario destaca el grupo de cuidados de la vivienda, telefonía, y energía con 34 por ciento, seguido de vestido y calzado y el de cuidados personales, con 18.5 y 18.4 por ciento, respectivamente.

Un aspecto que ayuda a explicar los menores niveles de pobreza e inseguridad alimentaria de San Salvador el Verde es su mayor capacidad para producir alimentos. Este tiene mejores condiciones agro-productivas, lo que implica una capacidad diferente para la producción de alimentos. San Salvador el Verde obtiene la mayor parte de ingreso agropecuario de la producción de hortalizas y granos, con un valor de la producción agrícola de 97 millones de pesos. Tlapanalá generó producción agrícola con valor de 73 millones y Coatzingo con 70 millones (SIAP, 2015).

\section{Variables relacionadas con la inseguridad alimentaria y la pobreza}

Las variables explicativas, que resultaron significativas, positivamente asociadas, en explicar la pobreza y la inseguridad alimentaria, fueron; el género del jefe(a) del hogar, hablar lengua indígena, jefe(a) del hogar con más de 60 años, dedicarse a una actividad productiva agropecuaria como actividad principal, sembrar una superficie de temporal menor a tres hectáreas, y tener cuatro o más dependientes del jefe(a). Las variables explicativas, asociadas negativamente con la pobreza e inseguridad alimentaria, son la escolaridad del jefe(a) del hogar nivel bachillerato o mayor, tener acceso a crédito, a seguridad social, dedicarse al comercio como actividad principal, y tener un índice de activos de 0.5 o mayor (Tabla 6).

El cambio en las probabilidades esperadas del género (ser jefa de hogar) sobre la condición de pobreza y sobre inseguridad alimentaria es de 0.37 y 0.48 , respectivamente. Lo que indica que ser jefa de hogar aumenta la probabilidad de ser pobre y tener inseguridad alimentaria en 37 y 48 por ciento, respectivamente. 


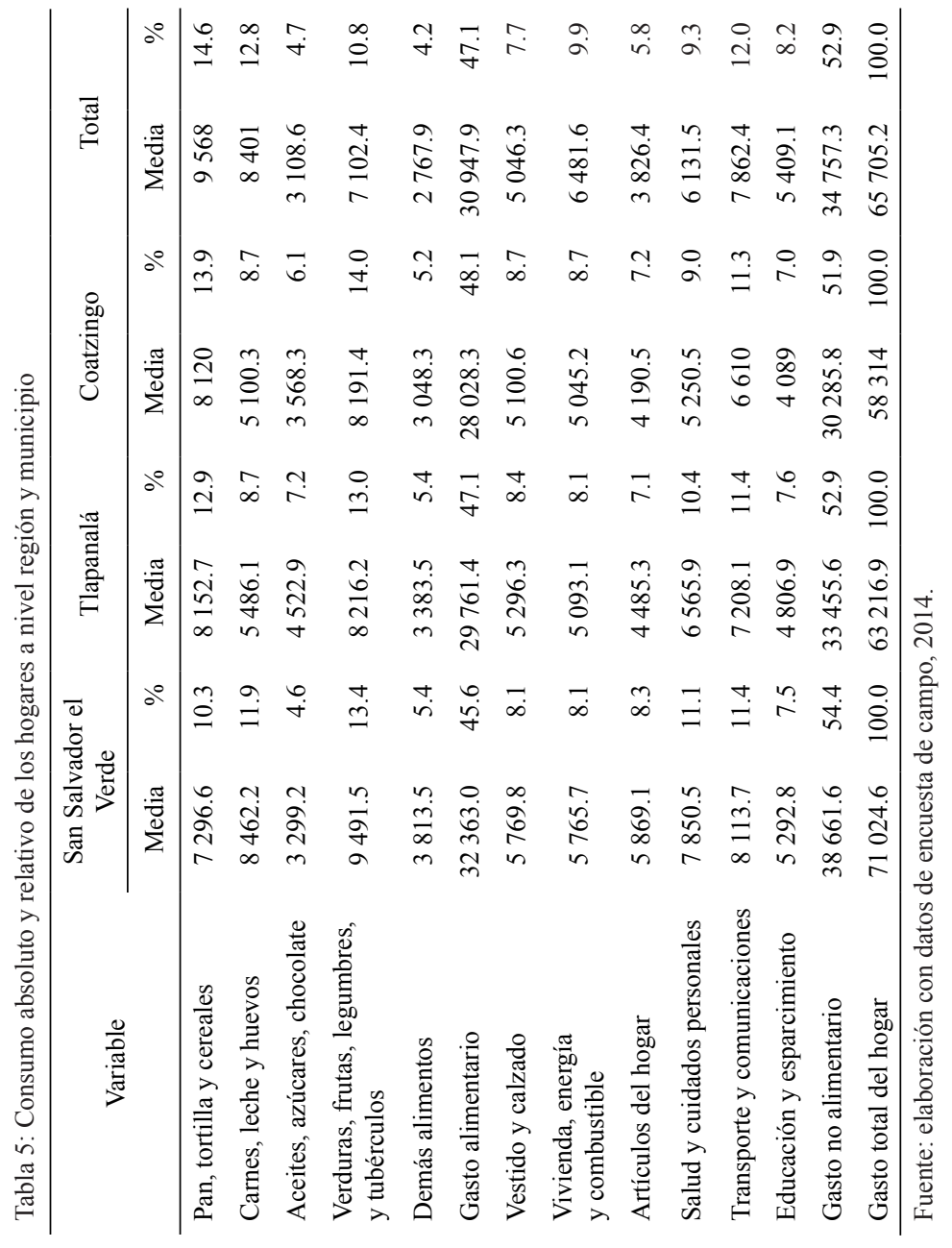


Factores relacionados con la pobreza, la inseguridad alimentaria y estrategias ... / J.L. JARAMILLO VILLANUEVA et al.

Tabla 6: Resultados de los modelos de inseguridad alimentaria y pobreza

\begin{tabular}{|c|c|c|c|c|c|c|}
\hline \multirow[b]{2}{*}{ Variable } & \multicolumn{3}{|c|}{ Inseguridad alimentaria } & \multicolumn{3}{|c|}{ Pobreza } \\
\hline & $\begin{array}{r}\text { Efectos } \\
\text { marginales }\end{array}$ & $\begin{array}{r}\text { Error } \\
\text { estándar }\end{array}$ & $\begin{array}{r}\text { Valor } \\
\text { de t }\end{array}$ & $\begin{array}{r}\text { Efectos } \\
\text { marginales }\end{array}$ & $\begin{array}{r}\text { Error } \\
\text { estándar }\end{array}$ & $\begin{array}{r}\text { Valor } \\
\text { de t }\end{array}$ \\
\hline Género & -0.8747 & 0.1055 & -8.29 & -0.3885 & 0.0933 & -4.17 \\
\hline Lengua indígena & 0.8349 & 0.1205 & -8.29 & -0.3133 & 0.0933 & -5.27 \\
\hline \multicolumn{7}{|l|}{ Edad del jefe (años) } \\
\hline Menor de 30 & 0.7540 & 0.0689 & 13.85 & 0.0525 & 0.0611 & 0.86 \\
\hline De 40 a 50 & 0.9381 & 0.0645 & 14.54 & 0.2792 & 0.1258 & 2.22 \\
\hline De 51 a 60 & 0.9139 & 0.0910 & 10.05 & 0.6240 & 0.1741 & 3.58 \\
\hline Mayor a 60 & -0.2347 & 0.2603 & -3.25 & -0.8524 & 0.1737 & -4.91 \\
\hline \multicolumn{7}{|l|}{ Escolaridad jefe(a) } \\
\hline Primaria & -0.9053 & 0.0985 & -9.19 & 0.3335 & 0.1036 & 1.22 \\
\hline Secundaria & 0.8222 & 0.1659 & 1.56 & 0.3539 & 0.1963 & 1.80 \\
\hline Preparatoria & 0.9887 & 0.0220 & 44.96 & 0.7887 & 0.0220 & 44.96 \\
\hline Profesional & 0.9832 & 0.7280 & 0.31 & 0.9232 & 0.5279 & 4.31 \\
\hline Transferencias asistenciales & -0.4179 & -0.0798 & -11.51 & -0.0630 & -0.0746 & -4.87 \\
\hline Recibió crédito productivo & 0.0762 & 0.0315 & 3.01 & 0.4371 & 0.2894 & 2.31 \\
\hline Seguridad social & 0.1297 & 0.0825 & 11.27 & 0.7507 & 0.3645 & 4.56 \\
\hline Recibió remesas & 0.3590 & 0.0680 & 3.58 & 0.0791 & 0.0128 & 2.18 \\
\hline \multicolumn{7}{|l|}{ Ocupación } \\
\hline Agropecuario & -0.9475 & 0.0531 & -17.86 & -0.4055 & 0.1472 & -2.75 \\
\hline Comercio & 0.7516 & 0.2026 & 3.71 & 0.4136 & 0.1702 & 2.43 \\
\hline Oficios & 0.8460 & 0.1469 & 5.76 & 0.1927 & 0.1086 & 1.77 \\
\hline Empleado & 0.8597 & 0.1465 & 5.87 & 0.4112 & 0.2350 & 1.75 \\
\hline \multicolumn{7}{|l|}{ Superficie sembrada } \\
\hline Hasta 1.0 ha. & 0.3641 & 0.0534 & 6.81 & -0.3500 & 0.0348 & -10.05 \\
\hline De 1.0 a 2.9 ha. & 0.7936 & 0.0961 & 8.26 & -0.3474 & 0.0338 & -10.29 \\
\hline De 3.0 a 4.9 ha. & 0.9610 & 0.0429 & 22.39 & 0.3448 & 0.0342 & 10.09 \\
\hline De 5.0 o más ha. & 0.9908 & 0.0196 & 50.59 & 0.3423 & 0.0359 & 9.53 \\
\hline \multicolumn{7}{|l|}{ Dependientes jefe(a) } \\
\hline De 1 a 2 dependientes & 0.4689 & 0.0572 & 8.20 & 0.3300 & 0.0341 & 9.69 \\
\hline De 3 a 4 dependientes & 0.4722 & 0.0360 & 13.12 & 0.4288 & 0.0719 & 5.97 \\
\hline Más de 4 dependientes & -0.2756 & 0.0839 & -5.67 & -0.5362 & 0.1482 & -3.62 \\
\hline Posee ganado mayor & 0.8927 & 0.0947 & 9.43 & 0.2153 & 0.0595 & 5.30 \\
\hline \multicolumn{7}{|l|}{ Activos productivos } \\
\hline De 1 a 2 activos & 0.4653 & 0.0412 & 11.31 & -0.3633 & 0.0473 & -7.68 \\
\hline De 3 a 4 activos & 0.4709 & 0.0328 & 14.35 & -0.3520 & 0.0352 & -10.01 \\
\hline De 5 a 6 activos & 0.4766 & 0.0389 & 12.24 & 0.8410 & 0.0350 & 9.75 \\
\hline Siete o más activos & 0.5823 & 0.0551 & 8.76 & 0.9302 & 0.0457 & 7.23 \\
\hline Eventos inesperados & -0.8850 & 0.1116 & -7.93 & 0.2375 & 0.0718 & 1.31 \\
\hline
\end{tabular}

Fuente: elaboración con datos de encuesta de campo, 2014. 
También puede observarse que ser jefa de hogar tiene un mayor efecto sobre la pobreza que sobre la inseguridad alimentaria. Si el jefe del hogar habla lengua indígena, la probabilidad de ser pobre y tener inseguridad alimentaria aumenta en 23 y 31 por ciento, respectivamente. La variable mujer indígena es altamente significativa; si se es jefa de hogar indígena aumenta la probabilidad en 58 y 65 por ciento.

Hogares encabezados por mujeres han sido especialmente identificados como de riesgo para experimentar malos resultados en seguridad durante crisis alimentarias (FAO, 2008). Scott y Diwakar (2016: 3) en un estudio en Bangladesh utilizando datos de panel, de 1997 a 2010, estudiaron los factores explicativos para salir de la pobreza de forma permanente versus escapes transitorios. Hogares encabezados por mujeres se identificaron con mayores probabilidades de sufrir un escape transitorio de la pobreza. Sin embargo, estos hogares cuando están asociados con remesas, activos y educación, mejoran de forma importante sus probabilidades de salir de la pobreza de forma permanente.

Se encontró una relación inversa entre la escolaridad del jefe y la condición de pobreza y en la de inseguridad alimentaria; a mayor nivel educativo disminuye la probabilidad de estar en condición de inseguridad alimentaria y de pobreza. Niveles altos de educación, principalmente nivel preparatoria o bachillerato, disminuyen la probabilidad de pobreza e inseguridad alimentaria en 26 y 55 por ciento respectivamente. Este resultado es consistente con lo reportado por Magaña-Lemus et al., (2016: 13), quienes encontraron que la probabilidad de tener seguridad alimentaria aumenta en 12.6, 20.2, y 28.4 por ciento si el jefe del hogar tiene escolaridad de secundaria, bachillerato, y profesional, comparado con educación primaria o menor. El efecto del nivel educativo es más significativo, y más intenso sobre la pobreza que sobre la inseguridad alimentaria. Resultados similares encontraron Scott y Diwakar (2016: 3). El nivel educativo, especialmente estudios post-secundarios (diez años o más) reducen de forma importante el riesgo de salir transitoriamente de la pobreza, debido a que mayores niveles de educación están asociados a empleos no agropecuarios, que generan ingreso más altos y permanentes. En este estudio se identificó que las variables empleo no agropecuario y niveles altos de educación del jefe del hogar están altamente correlacionados. Al respecto, Martínez (2015: 81) encontraron que las jefas de hogar con nivel bachillerato y licenciatura, en comparación con el grupo sin educación, aumentan su probabilidad de participar en actividades no agropecuarias en 20.55 por ciento y 46.84 por ciento, respectivamente. 
Factores relacionados con la pobreza, la inseguridad alimentaria y estrategias ... / J.L. JARAMILLO VILLANUEVA et al.

Las transferencias del gobierno, a través de programas asistenciales, son significativas, en explicar la situación de inseguridad alimentaria y la pobreza. Sin embargo, el efecto de esta variable sobre la inseguridad alimentaria es seis veces mayor que su efecto sobre la pobreza. El crédito es una variable significativa, no obstante, esta tiene un efecto importante en la pobreza, no así en la inseguridad alimentaria. Situación similar con el acceso a la seguridad social (acceso a servicios de salud del estado).

La posesión de activos productivos y ganado mayor disminuyen la probabilidad de tener inseguridad alimentaria y la condición de pobreza, en tanto que tener bajos niveles de activos, aumenta la probabilidad en 17.09 y 43.5 por ciento de tener inseguridad alimentaria y pobreza respectivamente. Scott y Diwakar (2016: 3) encontraron que los hogares con mayor valor de los activos son más propensos a experimentar un escape sostenido de la pobreza; ser propietario de tierras cultivables y cultivarlas reduce el riesgo de sufrir un escape transitorio de la pobreza en la medida que esto representa un componente importante del ingreso del hogar, debido a que además de los ingresos puede servir como garantía de préstamos. Un aumento en el número de animales se asocia con un menor riesgo de sufrir un escape transitorio de la pobreza debido al flujo de ingreso que generan durante todo el año. La tasa de dependencia está correlacionada negativamente con la pobreza. Los hogares que han sufrido dos a tres shocks en los últimos cinco años aumentan su probabilidad de permanecer en la pobreza.

\section{Estrategias de afrontamiento de la inseguridad alimentaria}

Los hogares rurales pobres con inseguridad alimentaria, en este estudio, practican diferentes estrategias de afrontamiento de su situación de pobreza y de inseguridad alimentaria, caracterizadas por la mezcla de actividades productivas agropecuarias y no agropecuarias, además de medidas de emergencia ante la inseguridad alimentaria extrema. La seguridad alimentaria o la inseguridad se pueden entender mejor estudiando las estrategias de afrontamiento que los hogares utilizan cuando no tienen acceso a los alimentos suficiente. Estas estrategias consisten en modificaciones de comportamiento, tales como comer alimentos que son menos preferidos, limitando tamaño de las porciones, y saltarse las comidas, entre otras (Ga1laher et al., 2013: 397).

Se encontró que las acciones más recurrentes son pedir alimentos prestados o donados a familiares ( 44.5 por ciento), pedir préstamos de dinero para comprar alimentos ( 35.6 por ciento) y compra de alimentos a crédito ( 27.8 por ciento). Las acciones más drásticas están relacionadas con la 
venta de activos de hogar como animales menores, mayores y herramientas (Tabla 7). Por municipio, y congruente con las condiciones de bienestar de cada municipio, en San Salvador el Verde los hogares realizan prácticas de mitigación de inseguridad alimentaria en menor proporción que los otros dos municipios.

Tabla 7: Estrategias de afrontamiento de la inseguridad alimentaria de corto plazo

\begin{tabular}{|c|c|c|c|c|}
\hline Estrategias o acciones & $\begin{array}{l}\text { San Salvador } \\
\text { el Verde }(\%)\end{array}$ & $\begin{array}{r}\text { Tlapanalá } \\
(\%)\end{array}$ & $\begin{array}{r}\text { Coatzingo } \\
(\%)\end{array}$ & $\begin{array}{r}\text { Total } \\
(\%)\end{array}$ \\
\hline $\begin{array}{l}\text { Alimentos prestados o donados } \\
\text { por familiares }\end{array}$ & 41.7 & 58.3 & 61.5 & 44.5 \\
\hline $\begin{array}{l}\text { Pidió dinero prestado a familiares } \\
\text { y vecinos }\end{array}$ & 32.3 & 63.9 & 65.7 & 35.6 \\
\hline Compró alimentos a crédito & 24 & 16.7 & 19.6 & 27.8 \\
\hline $\begin{array}{l}\text { Redujo el gasto en salud y } \\
\text { educativo }\end{array}$ & 19.8 & 22.2 & 20.2 & 20.5 \\
\hline Consumió las semillas de reserva & 14.7 & 27.8 & 32 & 16.9 \\
\hline $\begin{array}{l}\text { Adultos comieron menos } \\
\text { alimentos }\end{array}$ & 14.7 & 13.9 & 15.9 & 16.9 \\
\hline Vendió artículos del hogar & 17.7 & 8.3 & 12.5 & 15.9 \\
\hline $\begin{array}{l}\text { Vendió animales menores (cabras, } \\
\text { ovejas) }\end{array}$ & 7.9 & 8.1 & 38.1 & 10.8 \\
\hline Vendió aves de corral & 8.3 & 13.9 & 23.9 & 12.5 \\
\hline Vendió herramientas agrícolas & 6.3 & 4.6 & 6.8 & 6.5 \\
\hline $\begin{array}{l}\text { Vendió grandes animales (ganado, } \\
\text { burros) }\end{array}$ & 3.1 & 4.5 & 10.5 & 3.4 \\
\hline $\begin{array}{l}\text { Enviaron a los menores a vivir con } \\
\text { parientes }\end{array}$ & 1.4 & 2.7 & 4.7 & 2.2 \\
\hline
\end{tabular}

Fuente: elaboración con datos de encuesta de campo, 2014.

\section{Conclusiones}

Teórica y empíricamente, los niveles de ingreso y consumo están asociados con el nivel de pobreza de los hogares. Lo mismo puede decirse de los niveles de pobreza e inseguridad alimentaria. Los resultados encontrados están en esta dirección. Existe una correlación alta entre niveles de pobreza e inseguridad alimentaria. Los tres municipios del estudio presentan mayores niveles de pobreza e inseguridad alimentaria que los reportados por el CONEVAL. 
Observamos que, si bien la pobreza y la inseguridad alimentaria están altamente correlacionados, presentan rasgos que las hacen diferentes. Las variables explicativas de la pobreza son aquellas que tienen un carácter prolongado en sus efectos, como la educación, la posesión de activos, tener ganado mayor, tierra agrícola en cantidad mayor a cinco hectáreas, el empleo no agropecuario como actividad principal, recibir crédito productivo, y acceso a seguridad social. Las variables que mejor explican la inseguridad alimentaria parecen tener efectos de corto plazo en el consumo alimentario de las personas, como las transferencias asistenciales, la producción en pequeña escala para autoconsumo principalmente, y recibir remesas.

La pobreza y la inseguridad alimentaria en hogares rurales de la región de estudio está presente en porcentajes ligeramente mayores a los reportados por CONEVAL (2016). La pluriactividad como estrategia, es la característica principal para hacer frente a la pobreza y mantener niveles mínimos de bienestar. Las transferencias de gobierno y las remesas que reciben los hogares pobres juegan un papel importante en mitigar los efectos de la inseguridad alimentaria; representan la mitad del valor de la canasta no alimentaria de los hogares pobres.

Una característica central de los hogares rurales, en términos de su estrategia de sobrevivencia, es la diversificación del ingreso de fuentes como la agricultura, ganadería, venta de fuerza de trabajo, transferencias de gobierno, recepción de remesas y empleo no agropecuario tanto cuenta propia como salarial. Éstas últimas son cada vez más importantes, para lo cual el nivel educativo y el acceso a fuentes de empleo no agropecuario son fundamentales y debieran ser parte de una estrategia de superación de la pobreza.

El ingreso destinado al consumo de alimentos calculado es mayor al reportado por INEGI para áreas rurales, y se gasta principalmente en fuentes de carbohidratos, seguido de fuentes de proteínas. Lo que considerando que, en términos relativos, los carbohidratos son más baratos que la proteína, esto habla de una posible alimentación de mala calidad.

Una característica central de los hogares rurales, en términos de su estrategia de sobrevivencia, es la diversificación del ingreso de fuentes como la agricultura, ganadería, venta de fuerza de trabajo, transferencias de gobierno, recepción de remesas, y empleo no agropecuario tanto cuenta propia como salarial. Debido a la creciente importancia de este último al ingreso del hogar, las características y condiciones que favorecen este tipo de empleos deben fomentarse; básicamente mayor y mejor educación y acceso a fuentes de empleo. 


\section{REFERENCIAS BIBLIOGRÁFICAS}

Alkire, Sabina and Foster, James, 2011, "Counting and multidimensional poverty measurement", in Journal of Public Economics, vol. 95(7-8):476-487.

Barrett, Christopher B., 2010, "Measuring food insecurity", in Science, vol. 327(5967):825-828.

Barrett, Christopher B., 2013, Food security and sociopolitical stability, Oxford University Press, pp. 512.

Chinnakali, Palanivel, Ravi, Upadhyay, Deepa, Shokeen, Kavita, Singh, Manpreet, Kaur, Arvind, Singh and Chandrakant, Pandav S, 2014, "Prevalence of household-level food insecurity and its determinants in an urban resettlement colony in north India", in Journal of health, population, and nutrition, vol 32(2), 227.

Coates, Jennifer, Swindale, Anne and Bilinsky, Paula, 2007, "Household food insecurity access scale (HFIAS) for measurement of food access: Indicator guide", in Food and Nutrition Technical Assistance, USAID from the American People, pp. 1-32, disponible en http://www.fao.org/fileadmin/user_upload/eufao-fsi4dm/ doc-training/hfias.pdf

Coates, Jennifer, Frongillo, Edward, Lorge Rogers, Beatrice, Webb, Patrick, Wilde, Parke and Houser, Robert, 2006, "Commonalities in the experience of household food insecurity across cultures: What are measures missing?", in The Journal of Nutrition, vol. 136(5):1438S - 1448S.

CONAPO, 2012, Consejo Nacional de Población. México, consultado: 09/09/2016, disponible en http://www.conapo.gob.mx/en/CONAPO/Mexico_en_cifras

CONEVAL, 2016, Consejo Nacional de Evaluación de la Política de Desarrollo Social, México, consultado: 09/09/2016, disponible en http://www.coneval.org. $\mathrm{mx} /$ Medicion/Paginas/Evolucion_dimensiones_pobreza_1990_2015.aspx

De Janvry, Alain y Sadoulet, Elisabeth, 2001, "Income strategies among rural households in México: the role of Off-farm activities", in World Development, vol. 29(3):467-480.

FAO, 2006, Food and Agriculture Organization of the United Nations, consultado: 11/09/2016, disponible en http:/www.fao.org/docrep/014/al936s/ al936s00.pdf

FAO, 2008, "The state of food insecurity in the world 2008", in High food prices and food security-Threats and opportunities, Rome, FAO, pp. 56

FAO, 2011, Food and Agriculture Organization of the United Nations, consultado: 11/09/2016, disponible en http://www.fao.org/docrep/014/al936s/ al936s00.pdf

FAO, 2015, Food and Agriculture Organization of the United Nations, consultado: 11/09/2016, disponible en http://www.fao.org/countryprofiles/index/es/?iso3 $=$ MEX

Gallaher, C.M., Kerr, J.M., Njenga, M., Karanja, N.K. and Winklerprins, A.M.G.A., 2013, "Urban agriculture, social capital, and food security in the kibera 
Factores relacionados con la pobreza, la inseguridad alimentaria y estrategias ... / J.L. JARAMILLO VILLANUEVA et al.

slums of Nairobi, Kenya", in Journal of the Agriculture, Food, and Human Values Society, vol. 30(3):389-404.

Greene, William H., 2002, Econometric analysis, Prentice Hall, Upper Saddle River, New Jersey 07458, pp. 980.

Gundersen, Craig G. and Garasky, Steven, 2012, "Financial management skills are associated with food insecurity in a simple of households with children in the United States", in The Journal of Nutrition, vol. 142(10):1865-1870.

Harris-Fry, Helen, Azad, Kishwar; Kuddus, Abdul; Shaha, Sanjit; Nahar, Badrun; Hossen, Munir; Younes, Anthony Costello and Fottrell, Edward, 2015, "Socio-economic determinants of household food security and women's dietary diversity in rural Bangladesh: a cross-sectional study", in Journal of Health, Population and Nutrition, 33(1): 2.

Haughton, Jonathan E. and Khandker, Shahidur, 2009, Handbook on poverty + inequality, The World Bank, Washington, DC., pp. 419.

HLPE, 2011, Price volatility and food security, A report by the High Level Panel of Experts on Food Security and Nutrition of the Committee on World Food Security, Rome 2011, consultado: 11/09/2016, disponible en http://www.fao.org/, http://cuentame. inegi. org.mx/

Huerta, Quintanilla Rogelio, 2012, "Pobreza, distribución del ingreso y renta básica”, por Economía UNAM, vol. 9(26):68-81, UNAM, México.

INAFED, 2010, Enciclopedia de los municipios y delegaciones de México. Instituto Nacional para el Federalismo y el Desarrollo Municipal, Secretaría de Gobernación. Disponible en http://www.inafed.gob.mx/work/enciclopedia/EMM21 puebla/

INEGI, 2007, Censo Agrícola, Ganadero y Forestal, Instituto Nacional de Estadística y Geografía, consultado: 12/08/2016, disponible en http://www3.inegi.org. $\mathrm{mx} /$ sistemas/tabuladosbasicos/default.aspx? $\mathrm{c}=17177 \& \mathrm{~s}=\mathrm{est}$

INEGI, 2014, Censo de Población y Vivienda, Instituto Nacional de Estadística y Geografía, consultado: 11/09/2016, disponible en http://www.inegi.org.mx/est/ contenidos/proyectos/ ce/ce2014/

INEGI, 2015, Encuesta Nacional de Ingreso y Gasto de los Hogares, Instituto Nacional de Estadística y Geografía, consultado: 09/09/2016, disponible en http:// www.inegi.org.mx/est/contenidos/proyectos/encuestas/hogares/regulares/enigh/ default.aspx

Jobson, John D., 1992, Applied Multivariate Data Analysis, Springer Texts in Statistics, pp. 732.

Jonasson, Erik and Helfand, Steven, 2010, "How important are locational characteristics for rural non-agricultural employment? Lessons from Brazil", in World Development, vol. 38(5):727-741.

Lobos, Germán y Jean-Laurent, Viviani, 2008, "Factores determinantes de la utilización de instrumentos públicos para la gestión de riesgos en Chile: un modelo 
logit binomial", en Lecturas de Economía, vol. 69(2):63-83, Universidad de Antioquia, Colombia.

Magaña-Lemus, David, Ishdorj, Ariun; Rosson III, C. Parr; and Lara Álvarez, Jorge, 2016, "Determinants of household food insecurity in Mexico", in Agricultural and Food Economics, vol. 4(1):1-20.

Malik, Shahnawaz, 1996, "Determinants of rural poverty in Pakistan: A micro study", in The Pakistan development review, 35(2):171-187

Mango, Nelson, Byron, Zamasiya; Clifton, Makate; Kefasi, Nyikahadzoi; and Shephard, Siziba, 2014, "Factors influencing household food security among smallholder farmers in the Mudzi district of Zimbabwe", in Development Southern África, 31(4): 625-640.

Martínez, D. Marlen, 2015, El papel del empleo no agropecuario como estrategia de diversificación de ingresos en hogares rurales de México, Tesis de Doctorado, Colegio de Postgraduados Campus Puebla, México, pp.129.

Pedrero, Mercedes, 2004, "Género, trabajo doméstico y extra doméstico en México. Una estimación del valor económico del trabajo doméstico", en Estudios Demográficos y Urbanos, vol. 19:413-446.

Pinstrup-Andersen, Per, 2009, "Food security: definition and measurement", in Food Security, vol. 1(1):5-7.

Ponce-Sernicharo, Gabriela, 2012, "Vulnerabilidad social y riesgo de caer en pobreza en México", en Centro de estudios sociales y de opinión Pública, núm. 138:1-33, junio de 2012. México.

Rahut, Dil Bahadur y Micevska Scharf, Maja, 2012, "Livelihood diversification strategies in the Himalayas", in Australian Journal of Agricultural and Resource Economics, vol. 56(4):558-582.

Ravallion, Martin and Jyotsna, Jalan, 1998, Determinants of transient and chronic poverty: evidence from rural China. The World Bank.

Sadoulet, Elisabeth y Janvry, Alain de, 1994, "Quantitative development policy analysis”. Baltimore, MD: experience. Food Policy, vol. 25:447-462.

Scarnecchia, D. L. and Gaskins, C.T., 1987, "Modeling animal unit equivalents for beef cattle", in Agricutural Systems, vol. 23(1):19-26.

Schwentesius, Rita Rindermann y Ayala Garay, Alma Velia, 2014, Seguridad y soberanía alimentaria en México. Análisis y propuestas de Política, Plaza y Valdés, México, pp.196.

Scott, Lucy and Diwakar, Vidya, 2016, "Ensuring escapes from poverty are sustained in Uganda", USAID, Leveraging Economic Opportunities report num. 27, pp. 60, consultado: 22/09/2016, disponible en https://www.microlinks.org/sites/ default/files/resource/files/ Transitory_escapes_and_Resilience_UgandaCaseStudy_final_edited_LS_002_....pdf

Sen, Amartya, 1987, The standard of living, Cambridge, Cambridge University Press, pp. 140. 
Factores relacionados con la pobreza, la inseguridad alimentaria y estrategias ... / J.L. JARAMILLO VILLANUEVA et al.

Sen, Amartya, 1992, Inequality reexamined, Harvard University Press (1995), pp. 224.

SIAP, 2015, Servicio de Información Agroalimentaria y Pesquera, 2015, consultado: 09/09/2016, disponible en http://infosiap.siap.gob.mx:8080/agricola_siap_ gobmx/ ResumenDelegacion.do

Urquia-Fernandez, Nuria, 2014, "La seguridad alimentaria en México", in Food securrity in Mexico 2014; 56 suppl 1: S92-S98.

Valdés, Guadalupe, 1998, "The world outside and inside schools: Language and immigrant children", in American Educational Research Association, vol. 27(6):418 ,

Wang Xiaolin, Hexia, Feng; Qingjie, Xia; and Sabina, Alkire, 2016, On the relationship between income poverty and multidimensional poverty in China, OPHI Working Paper 101, University of Oxford, pp 1-21.

Yúnez-Naude, Antonio and Taylor, Edward, 2001, "The determinants of nonfarm activities and incomes of rural households in Mexico, with emphasis on education”, in World Development, 29(3): 561-572.

Yúnez-Naude, Antonio y Taylor, Edward, 2004, "Los determinantes de las actividades y el ingreso no agrícola de los hogares rurales de México, con énfasis en la educación", Empleo e ingresos rurales no agrícolas en América Latina, CEPAL seminarios y conferencias núm. 35:231-244, Santiago de Chile.

\section{RESUMEN CURRICULAR DE LOS AUTORES}

\section{José Luis Jaramillo Villanueva}

El Dr. José Luis Jaramillo Villanueva obtuvo el grado de ingeniero agrónomo por la Universidad Autónoma Agraria Antonio Narro en 1990, el de maestro en ciencias en Economía Agrícola por el Colegio de Posgraduados en 1995 y el de Doctorado por la University of Guelph, en Ontario Canadá en 2006. Este último con especializaciones en mercados agroalimentarios y Políticas públicas. Actualmente es profesor-investigador titular del Colegio de Posgraduados en Ciencias Agrícolas, investigador Nacional nivel 1, e imparte el curso de Economía Agroalimentaria. Actualmente dirige la línea de investigación en Economía del Desarrollo Agrícola y Rural, del posgrado en Estrategias en Desarrollo Agrícola Regional del Colegio de Posgraduados. Ha publicado más de 30 artículos científicos, tres libros y más de diez capítulos de libro. Es arbitro de tres revistas científicas y consultor internacional.

Dirección electrónica: jaramillo@colpos.mx

Registro ORCID: http://orcid.org/0000-0001-8179-6351 


\section{Venkatesh Gurusamy}

El Dr. Venkatesh Gurusamy obtuvo su licenciatura en agricultura en el Agricultural College and Research Institute, en Madurai, Tamil Nadu en el año 2000, su maestría en Ciencias en Economía Agrícola en la misma institución y el doctorado en el Colegio de Posgraduados en Ciencias Agrícolas en el posgrado en Estrategias para el Desarrollo Agrícola Regional, en 2015. Actualmente es investigador en el JKK Munirajah College of Agricultural Science como profesor asistente (desde febrero del 2016). Sus líneas de investigación son "Bienestar en el medio rural" y "Economía de la producción agropecuaria".

Dirección electrónica: venil2@hotmail.com

Registro ORCID: http://orcid.org/0000-0002-5323-3647

\section{Leobardo Jiménez Sánchez}

Ingeniero Agrónomo egresado de la Escuela Nacional de Agricultura (ENA) donde obtuvo el título de Ingeniero Agrónomo, Especialista en Fitotecnia en 1960. Los grados de maestría y doctorado en ciencias los obtuvo en la Universidad de Wisconsin, Madison. Es Profesor Investigador Emérito del Colegio de Postgraduados e Investigador Nacional Emérito del Sistema Nacional de Investigadores. Su área de investigación es Desarrollo Rural Sustentable con las líneas "Estrategias para el Desarrollo Agrícola Regional” y "Evolución de la Educación, la Investigación y la Vinculación Agrícolas en México". Sus publicaciones recientes son “Análisis de producción y comercialización de hortalizas: caso del mercado de Huixcolotla, Puebla", en Revista Mexicana de Ciencias Agrícolas, vol. 5. núm. 4, 16 de mayo - 29 de junio, 2014 p. 687-694. Ensayos escogidos. Editorial Colegio de Postgraduados. ISBN: 978-607-715-230-9. "La agricultura mexicana y su potencial en la alimentación: el contexto actual", en Ciencia, Tecnología e Innovación en el Sistema Agroalimentario de México. Editorial Colegio de Postgraduados-AM-CONACYT-UPAEP-IMINAP, San Luis Huexotla, Texcoco, México. ISBN: 978-607-715-314-6.

Dirección electrónica: ljs@colpos.mx

Registro ORCID: https://orcid.org/0000-0003-1019-442X

\section{Daniel Claudio Martínez Carrera}

Profesor investigador titular del Colegio de Postgraduados, donde dirige la línea de generación y aplicación de conocimiento sobre biotecnología de hongos comestibles, funcionales y medicinales desde 1989, con el financiamiento de proyectos nacionales e internacionales. Cuenta con experien- 
cia en docencia y formación de recursos humanos de alto nivel, a través de la coordinación de programas de posgrado (1997-2016), la impartición de cursos de posgrado (Biotecnología y Desarrollo Sostenible), así como la dirección de tesis de maestría y doctorado de nivel nacional e internacional. Ha recibido diversas distinciones académicas nacionales e internacionales (e.g., Premio Nacional en Ciencia y Tecnología de Alimentos 1992, México; The IFS/King Baudouin Award 1992, Estocolmo, Suecia; Premio Colegio de Postgraduados a la Investigación y Desarrollo Tecnológico 2011, México; Premio Cuezcomate a la Innovación y Transferencia Tecnológica UAEM 2014, México). Coeditor fundador de la revista científica Micologia Aplicada International (www.micaplint.com). Fundador y coordinador de la Red Latinoamericana de Hongos Comestibles y Medicinales (www. hongoscomestiblesymedicinales.com). Ha publicado más de 113 artículos en revistas científicas nacionales e internacionales, así como tres patentes. Dirección electrónica: dcarrera@colpos.mx Registro ORCID: https://orcid.org/0000-0003-3878-1802 